\title{
THE PROTOTYPE VERY LONG BASELINE AMPLITUDE INTERFEROMETER
}

\section{Kurt M. Liewer}

Department of Physics and Astronomy, University of Maryland, College Park, Maryland, 20742, USA

\section{ABSTRACT}

An Amplitude Interferometer based on the design of an earlier interferometer but with two independent telescopes has been built at Maryland. The design and the current status of the instrument will be discussed.

This interferometer has been built with automatic guiding in each arm and is intended for use with baselines of 50 meters but can be used with longer baselines at restricted declinations.

The visibility detection will be exactly the same as that used in the cassegrain model of the interferometer which has operated successfully for several years on the 100 and 200 inch Hale telescopes. The visibility will be measured simultaneous1y in three different paths with slightly different delays which will allow for automatic location and tracking of the white light fringe envelope.

\section{INTRODUCTION}

Previous work at the University of Maryland ${ }^{1-8}$ with a cassegrain mounted Amplitude Interferometer has shown the principles used in its construction to work we11. It has been used to measure the diameters of 19 stars, 9 of them previously unmeasured and with an accuracy of .003 arc seconds or better in most cases $^{8}$.

The resolution of this instrument is limited by the size of the largest available telescope (5m). The desire for more resolution can be satisfied however by bringing light to the interferometer not from the edges of a single large mirror but from two small independent mirrors.

This paper will describe the long baseline Amplitude Interferometer, which has been built at the University of Maryland. Both its basic operating 
principles and 1ts current status will be discussed. Although the instrument has been called a prototype, it has been designed so that after local system tests it can be relatively easily transported to a better permanent observing site where astronomically useful observations can be carried out at baselines up to 50 meters. It is also expected to carry out limited tests at baselines up to 1000 meters in length.

\section{THE CASSEGRAIN INTERFEROMETER}

An Amplitude Interferometer built at Maryland which operates at the cassegrain focus of a large telescope has been in use for six years. This interferometer gives reference star visibilities in the range 0.6 . to 0.9 depending on the seeing and diameters measured with this instrument have errors of $\sim .003$ arc seconds as determined by internal consistency. 7,8

Because of the success of this interferometer, the long baseline interferometer has incorporated many of the design features of the cassegrain interferometer; one of the most important being the fringe detection method. Because of the similarities, the simpler cassegrain instrument will be briefly described first along with a simplified analysis of the detection scheme.

The cassegrain interferometer ${ }^{7}$ shown in Figure 1 operates as follows:

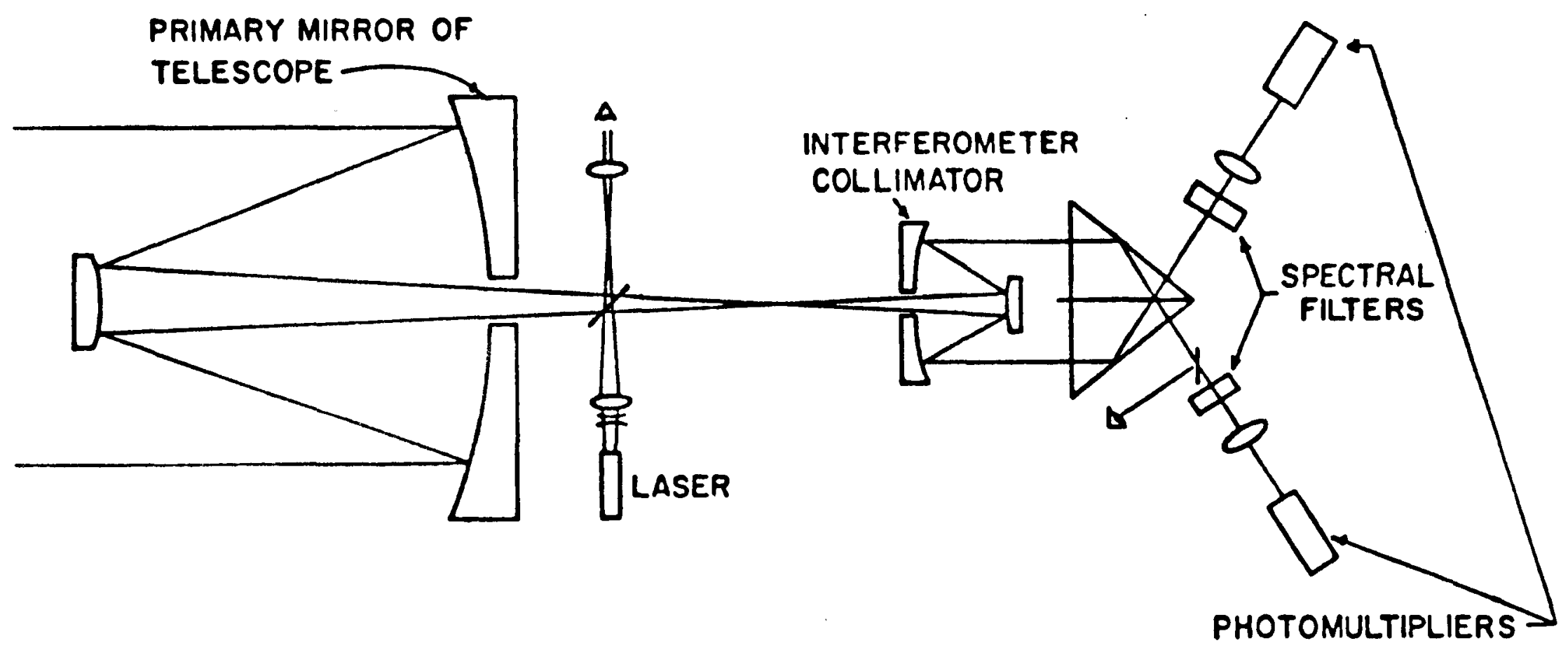

Figure 1

A schematic drawing of the cassegrain mounted model of the Amplitude Interferometer 
Light from the telescope is collimated and a mask containing two apertures is placed in the reduced beam, the projection of these apertures on the telescope primary mirror defines the instrument's baseline. The 1ight from these apertures then enters a Köster prism, which consists of an equilateral triangle with a beamsplitter in the center. This prism divides the light from each aperture and interferes it with the light from the other aperture. The prism is aligned such that the light interferes in a flat field, that is, no spatial fringes are produced in the output beams. The output beam intensity does change however as the relative phase of the two beams changes due to atmospheric turbulence. This system is very easy to use and has high efficiency. Only a single photomultiplier is needed and no fringe mask is required. The köster prism is used rather than separate mirrors and beamsplitsince it freezes most of the interferometrically important surfaces into a single glass piece so that their alignment is always correct.

A small laser is used to align the system including setting the prism to give flat field fringes. Once this is done, superimposing the laser and star at focus is sufficient to guarantee that the starlight will also interfer with a flat field.

As mentioned previously the output of the Köster prism does vary in intensity as the relative phase of the inputs is varied. A measurement of the depth of the intensity modulation would then be sufficient to determine the fringe visibility. This measurement is complicated by the scintillation or intensity variations in the input beams which is relatively large. Another property of the Köster prism is used to eliminate the scintillation effects; namely, the interference effects in the two output beams are out of phase. This is easily seen for the case of a non-absorbing beamsplitter where, by energy conservation, when one output beam is bright the other must be dim. Any change in brightness of an input beam however will cause both outputs to change in the same direction.

If $I_{1}(t)$ and $I_{2}(t)$ are the output beam intensities at time $t$ then they can be expressed as 


$$
\begin{aligned}
& I_{1}(t)=I_{0}(1+S(t)+V \sin \phi(t)) \\
& I_{2}(t)=I_{0}(1+S(t)-V \sin \phi(t))
\end{aligned}
$$

Here $S$ is a fluctuating term due to scintillation and $V \sin \phi(t)$ is due to interference, where, by definition, the time averages of $S$ and $V \sin \phi(t)$ $\left(\langle S\rangle_{T},\langle V \text { sin } \phi(t)\rangle_{T}\right)$ are zero. The quantity $\phi(t)$ is the relative phase of the light at the two apertures which the atmosphere will cause to vary by many radians.

The auto (AC) and cross (CC) correlations of the two beams are given by

$$
\begin{aligned}
& A C=I_{1} \cdot I_{1}+I_{2} \cdot I_{2}=2 I_{0}^{2}\left(1+2 S+S^{2}+V^{2} \sin ^{2} \phi(t)\right) \\
& C C=I_{1} \cdot I_{2}+I_{2} \cdot I_{1}=2 I_{0}^{2}\left(1+2 S+S^{2}-V^{2} \sin ^{2} \phi(t)\right)
\end{aligned}
$$

using $\langle S\rangle_{T}=0$ and $\left\langle\mathrm{V}^{2} \sin ^{2} \phi(\mathrm{t})\right\rangle_{\mathrm{T}}=\frac{\mathrm{V}^{2}}{2}$ their averages are

$$
\begin{aligned}
& \langle\mathrm{AC}\rangle_{\mathrm{T}}=2 \mathrm{I}_{0}^{2}\left(1+<\mathrm{S}^{2}>+\frac{\mathrm{V}^{2}}{2}\right) \\
& \langle\mathrm{CC}\rangle_{\mathrm{T}}=2 \mathrm{I}_{0}^{2}\left(1+<\mathrm{S}^{2}>-\frac{\mathrm{V}^{2}}{2}\right)
\end{aligned}
$$

Using Michelson's definition of the visibility

$$
|\gamma|=\frac{I_{\text {max }}-I_{\text {min }}}{I_{\text {max }}+I_{\min }} \text { we Identify the quantity } V \text { as the }
$$

visibility and may write

$$
|\gamma|^{2}=\frac{\langle\mathrm{AC}\rangle-\langle\mathrm{CC}\rangle}{\mathrm{I}_{0}} \quad\left\langle\mathrm{~S}^{2}\right\rangle=\frac{\langle\mathrm{AC}\rangle+\langle\mathrm{CC}\rangle}{2 \mathrm{I}_{0}}
$$

In forming the correlations $\mathrm{AC}$ and $\mathrm{CC}$ it is important that they be measured in a time shorter than the time for the atmosphere to change or the values of $S$ and $V$ must be replaced by $\langle V\rangle_{T}$ and $\langle S\rangle_{T}$ which are zero and $\mathrm{AC}=\mathrm{CC}=2 \mathrm{I}_{0}^{2}$, a result of little value.

In this instrument the number of photons arriving in one channel in an atmospheric correlation time is generally less than one so the correlations are done by digital rather than by analog means. The effectiveness of this detection scheme will be discussed in Section $V$. 


\section{THE LONG BASELINE INTERFEROMETER}

The long baseline interferometer is constructed by feeding 1ight from two separated mirrors, rather than one large mirror, to an interferometer such as the one just described.

The mirrors are arranged for convenience as shown in Figure 2. The reason this is conventent is that the phase delay in the one arm is a constant and so the compensating delay line need not be driven. The actual baseline configuration used for tests at the Maryland site has a short horizontal section between the two inner bending mirrors. The result is that the delay line must be driven as a function of time but only at a reasonably slow rate.

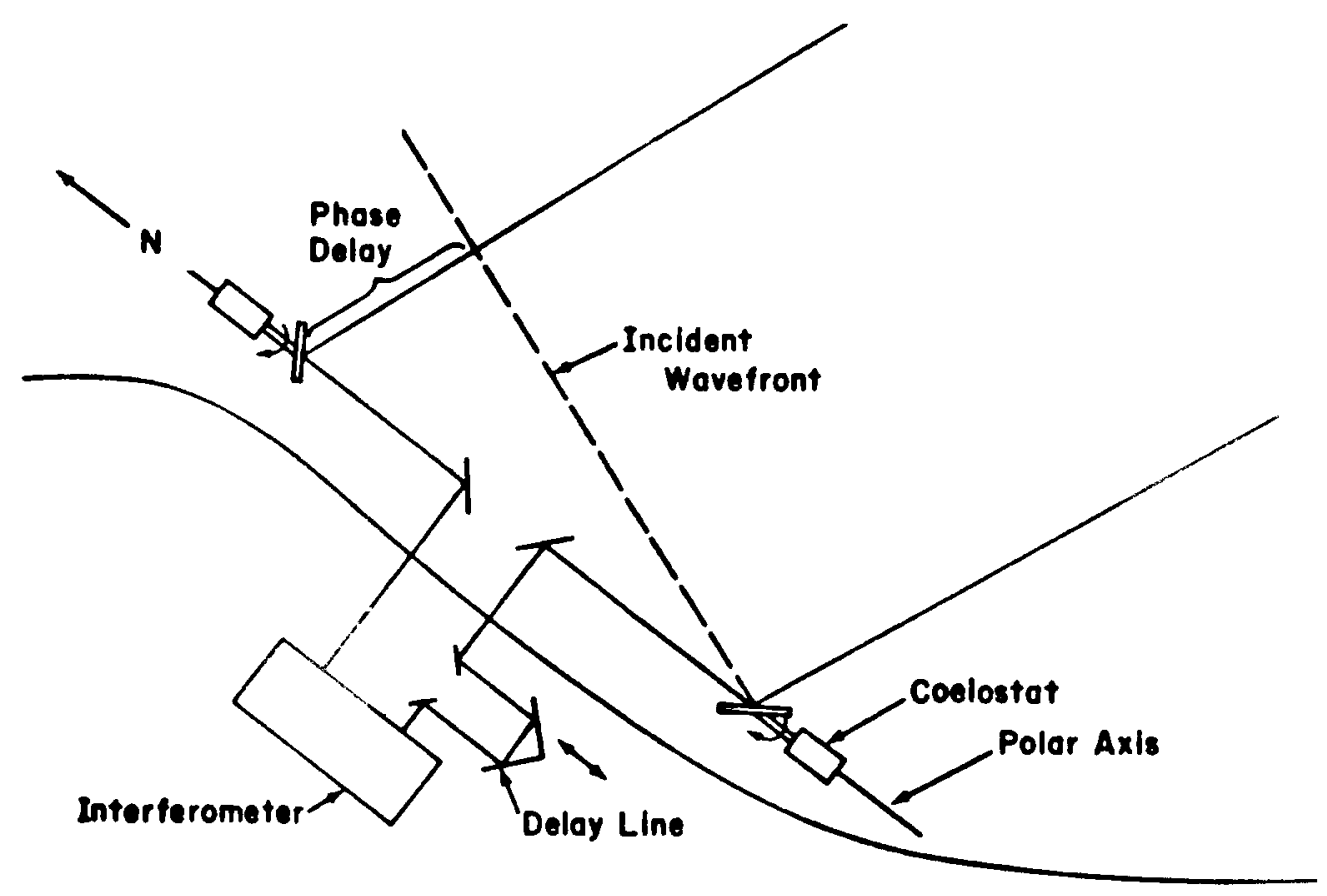

Figure 2

Configuration of Amplitude Interferometer with separate mirrors. With the baseline parallel to the polar axis the delay line position does not change as function of Local Hour Angle.

Figure 3 shows the outline of the actual interferometer. Basically, the starlight is split by a polarizing beamsplitter (only a single polarization may be used by the prism) and the part which will not be interfered is sent to both an eyepiece and to a quadrant detector whose signal will be used to stabilize the image. The light to be interfered is collimated and sent into the delay line which consists of corner retroreflectors. In being reflected 


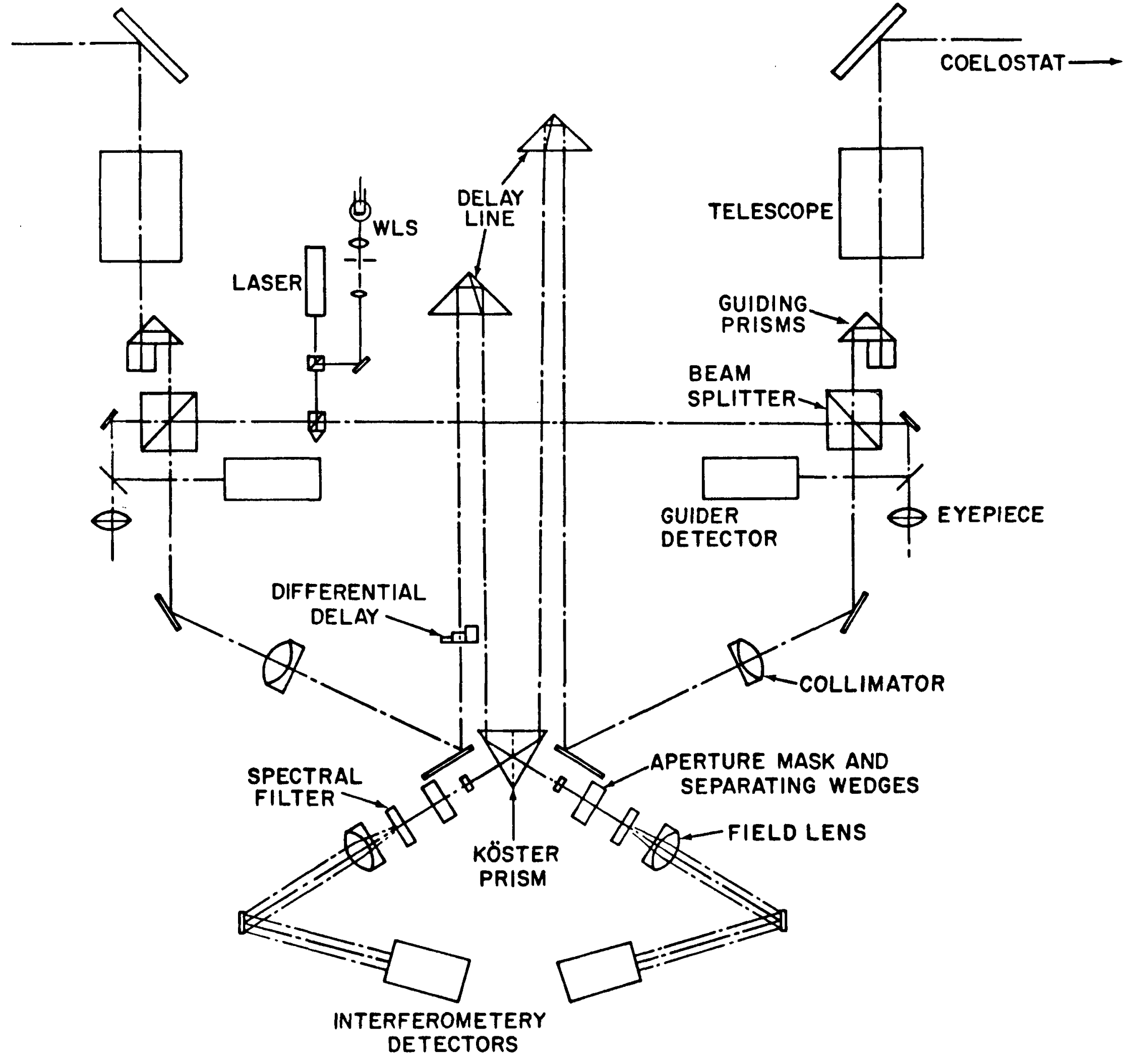

Figure 3

Schematic layout of the long baseline Amplitude Interferometer 
the beams are also translated so that they then enter the Köster prism.

One leg of the delay line can be moved to a number of fixed positions to make gross changes in the relative delay while the other side is moved by means of a precision screw driven by a DC motor coupled to an optical encoder. The motor rate is controlled by a minicomputer.

A white light source and a small laser provide a means for aligning the interferometer and the central fringe of the white light source gives an accurate calibration point for the relative delay.

The interferometer components are assmebled on a four foot by eight foot honeycomb optical table which is internally damped so that vibration problems are minimized.

Several problems occur in this interferometer which were not present in the cassegrain model.

The first of these is that the two arms must be guided independently and in doing so no delays can be introduced by the guiding mechanism. The method we have chosen is shown in Figure 4a. Figure $4 \mathrm{~b}$ shows the operation in a single axis. The motion of the prism causes a beam translation but does not change the path length. In the interferometer a pair of prisms cemented together are driven in the two axis perpendicular to the beam by means of two sma11 DC motors moving translation stages.
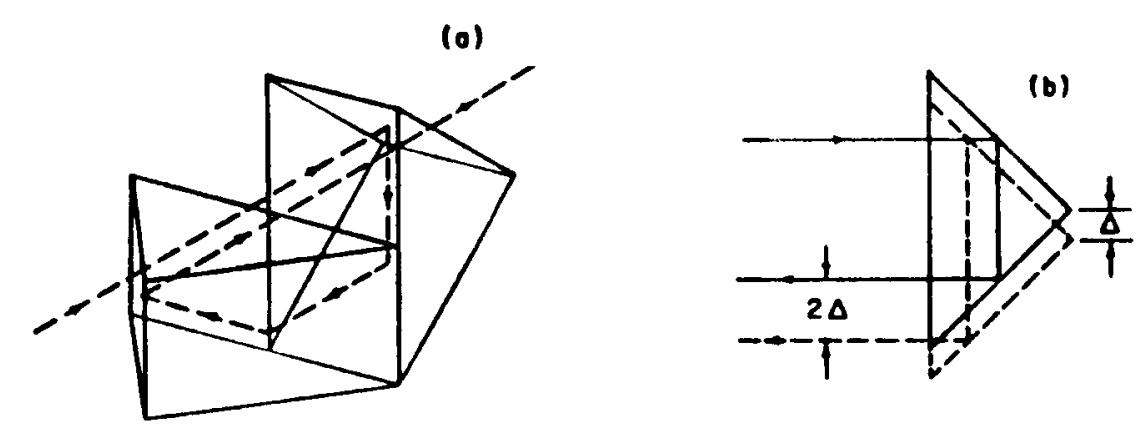

Figure 4

a) Constant phase prism translator and light path

b) Single axis operation and change in 1ight path with change in position 
A second new problem is that of locating the white light fringe for a stellar source. The method to be used is shown schematically in Figure 5 . Here, the fringe visibility is modulated by an envelope whose width depends on the spectral bandpass used. In the interferometer, three interferometers are actually run simultaneously, each having a slightly different delay When the visibility has been measured in three channels (Figure 5b) these values can be used to alter the delay line position until the channel with intermediate delay is at the positon of peak visibility (Figure $5 \mathrm{c}$ ). The three delays are introduced into the channels by means of two small pieces of glass inserted into the beam before the Köster prism. The three channels are then separated by means two weak prisms and then focussed onto a quadrant detector which serves as the detector for all three channels.

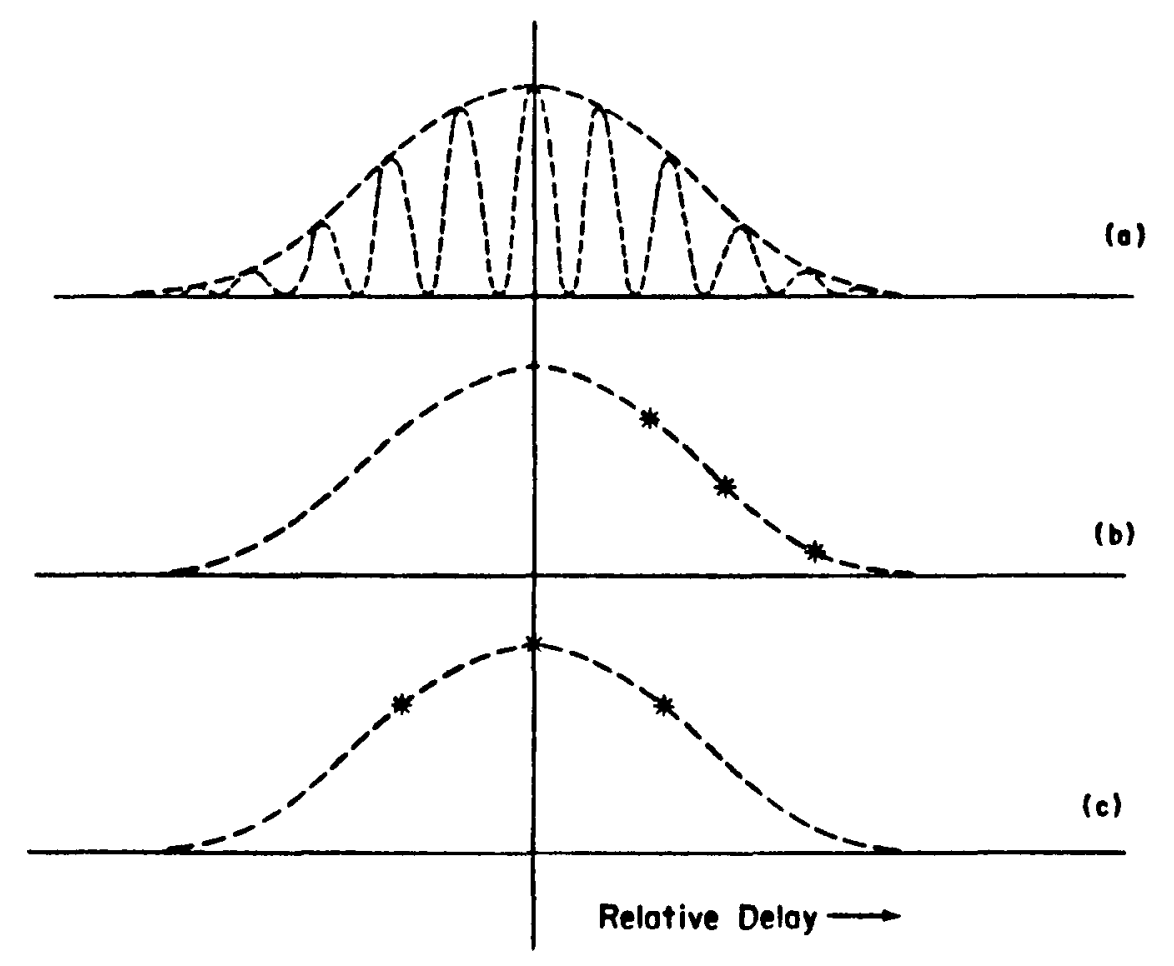

Figure 5

a) Modulation of fringe envelope due to spectral bandpass

b) Visibility measurements are made at three different delays

c) The delay line is moved to place the intermediate delay at the zero fringe

The difference in the optical path length in the two arms of the interferometer must be within a few wavelengths of the correct value or the visibility measurements will be degraded and for some of the geophysical problems the tolerence is reduced to a fraction of a wavelength. To monitor the path 
length a distance measuring laser interferometer is used. The beams from this interferometer are inserted into the stellar interferometer after the the Köster prism at a point near the aperture masks and separating wedges. They are split by the Köster prism and travel out through the optics to the coelostat mirrors where they are reflected by retroreflectors back into the system and are eventually recombined by the Köster prism. Any change in the relative lengths of the arms is then detected.

Because of their unique design and extensive use in this interferometer the quadrant detectors need to be described more fully. Figure 6a shows the configuration of the tube. In operation photoelectrons from a spot on the photocathode are accelerated to $20 \mathrm{Kev}$ and focussed by means of an electrostatic lens onto a corresponding spot on a silicon quadrant diode array. Each photoelectron produces about 2000 electron-hole pairs in the silicon as it is brought to a stop. This signal is large enough to easily do single photoelectron discrimination. The actual diode array dimensions are shown in Figure 6b. These tubes have been used for several years to do automatic guiding on the cassegrain interferometer. One of their best features is their compactness. The entire assembly including preamps is only two inches in diameter and six inches long.

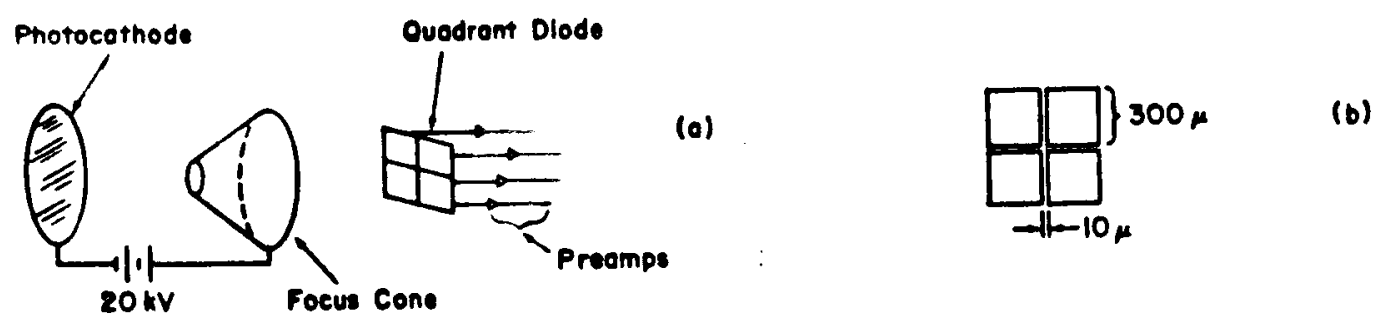

Figure 6
a) Schematic diagram of photon counting quadrant phototube
b) Actual dimensions of diode array

\section{CURRENT STATUS OF THE LONG BASELINE INTERFEROMETER}

The interferometer is set up at this time at a test site on the grounds of the optical research facility of the Goddard Space Flight Center. The baseline used is slightly less than three meters. Fabrication is complete 
except for the electronic fringe detectors and the insertion of the laser beams of the distance measuring interferometer. Visual fringes were looked for on several nights but not seen. Visual observation of fringes (in the aperture plane) at this site has proved difficult in the past although they were easily detected electronically with the cassegrain interferometer. Measurements with the cassegrain interferometer show that, at least at moderate baselines, the atmosphere will not be a serious problem.

Several other problems which are unique to this instrument have been investigated and are described below.

Vibration of the optical components is a possible cause for worry particularly because there are noise sources (delay line and guider motors) on the optical table. Accelerometer measurements show that for frequencies greater than 50 Hertz the table vibrations (with motors running) will not affect visibility measurements. Visual observations of fringes produced by the laser or white light source on the table show no motion.

Vibrations of the coelostat mirrors could also be a problem and for geophysical measurements the absolute positions of the mirrors is very important. Measurements with the distance measuring interferometer show no indication of vibration. Mirror motion due to bearing runout has been measured along the Right Ascension axis and upper limits placed on the motion in the perpendicular plane. Neither motion is large enough to affect the visibility measurements and will only have minimal effect on astrometric (or geophysical) measurements.

Finally, the total light path that is near the ground is relatively long and the path does go from an outside environment to inside a closed trailer which protects the interferometer and it is possible that the turbulence encountered in this part of the path could be excessive. To test this light was sent from a position behind the köster prism (at the detector lens focus) out through the interferometer to the coelostats which were pointed so that the light passed back through the optics a second time and finally was viewed at the focus of the opposite detector lens. Although the turbulence did move the fringes about they were easily seen visually. 


\section{MEASUREMENTS WITH THE CASSEGRAIN INTERFEROMETER}

Spatial interferometers such as those described in this paper are of interest because the atmospheric turbulence prevents the high angular resolution data they produce from being obtained in any other way. A very important question is what effect will the atmosphere have on the long baseline interferometer. At baselines greater than 5 meters there are no direct measurements of what the atmospheric effects will be. However, observations with the cassegrain interferometer at baselines up to 5 meters show no indication of any significant degradation in measured visibility with increasing baseline. ${ }^{7,8}$ The results obtained with the cassegrain interferometer also show the stability and quality of the data that can be obtained with the detection system already described.

Figures $7 \mathrm{a}$ and $7 \mathrm{~b}$ are plots of uncorrected visibilities measured at the Mt. Wilson Observatory 100-inch telescope on two different occassions. The sources were unresolved reference stars and the visibilities measured run from 0.7 to 0.9 . In the design of the interferometer, filter widths and aperture sizes were chosen as a compromise between sensitivity to changing atmospheric effects and limiting magnitude. The filter widths are about $100 \AA$ and the apertures are 4 centimeters in diameter.
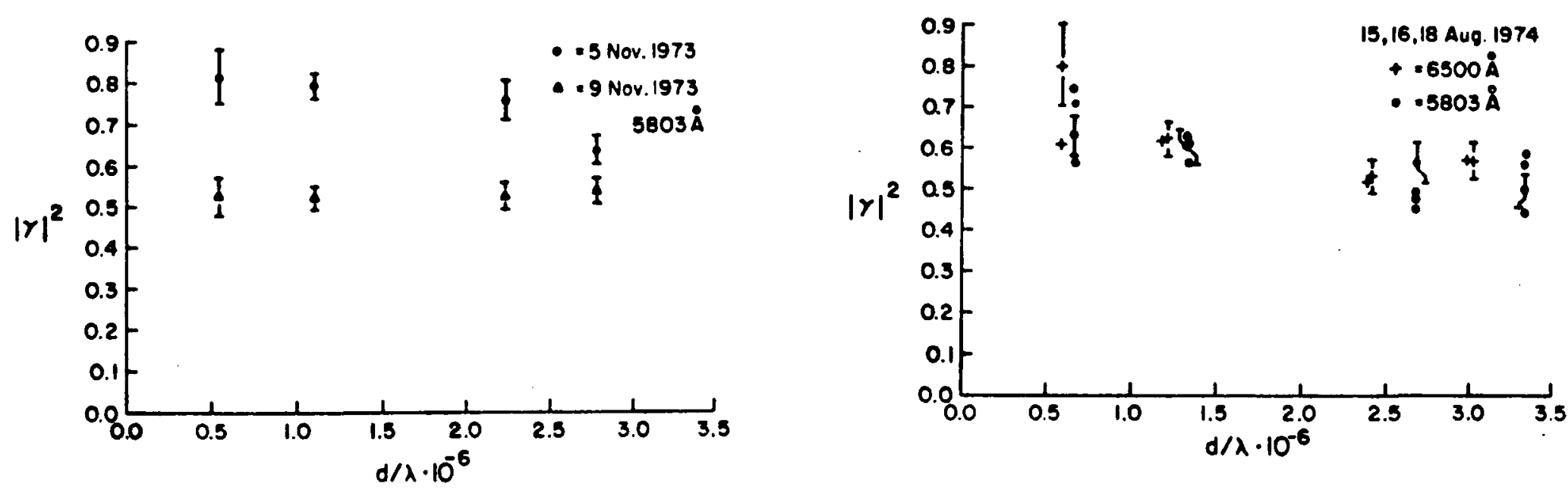

Figure 7

The measured uncorrected visibility squared for reference stars is shown as a function of spacing. Both sets of data are from the Mt. Wilson 100" telescope. The set taken in November is typical of day to day variations. 
Calculations by D. G. Currie here at Maryland indicate that with these parameters the atmospheric degradation of the visibility ( with 2 arc second seeing) should be about $20 \%$, in reasonable agreement with the observations. The long baseline interferometer will use similar filter widths and apertures.

The question of whether reasonable visibility measurements can be made with a long baseline amplitude interferometer is addressed by the data shown in Figures $8 \mathrm{a}$ and $8 \mathrm{~b}$. Here the visibilities of unresolved stars are plotted versus aperture spacing. Each point is the average of several nights observations which have been normalized by the visibility measured at the second separation. This is done to remove the night to night variation in the absolute value of the measured visibility.
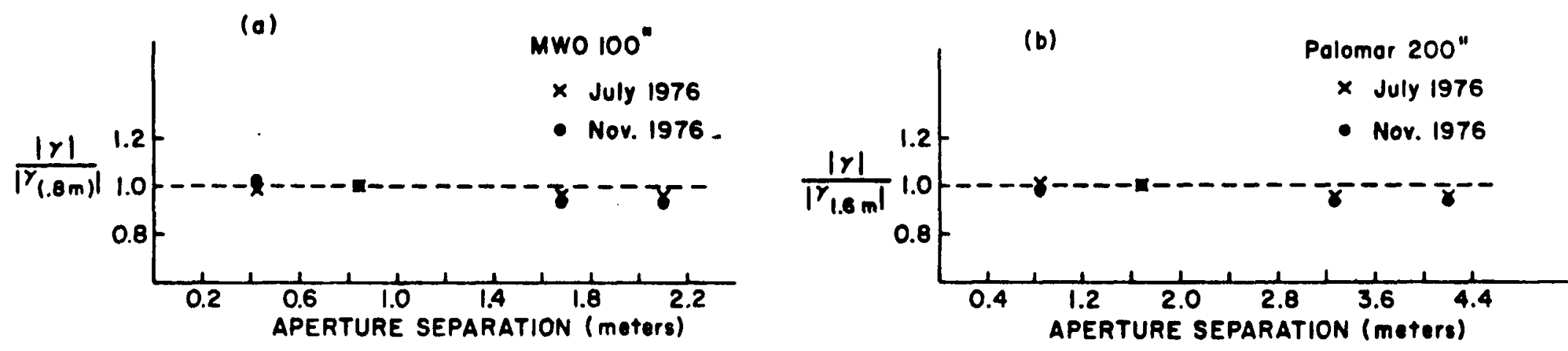

Figure 8

Average normalized visibility of unresolved stars as a function of aperture spacing. The small decrease in visibility with increasing separation is explained by increased sensitivity to guiding errors.

The resulting points show very little fall off with increasing separation. What reduction there is, is explained by instrumental variations from spacing to spacing and more important by increased sensitivity to guiding errors with increased separation. This later effect will not be present in the long baseIine interferometer as the phase delay does not change when the coelostats are misguided. The very small decrease in visibility with increasing separation indicates that up to 50 meters any decrease in visibility would be easily treated by simple normalization with reference star measurements.

Finally, the stability of the measurements made by the technique of amplitude interferometry is demonstrated in Figure 9. Observations of the star a Orionis have been made for five years with the cassegrain interferometer. 
No deviation from a constant diameter of $52.6 \mathrm{milliarc-seconds} \mathrm{is} \mathrm{discernable}$ to the level of rough1y $1 \%$.

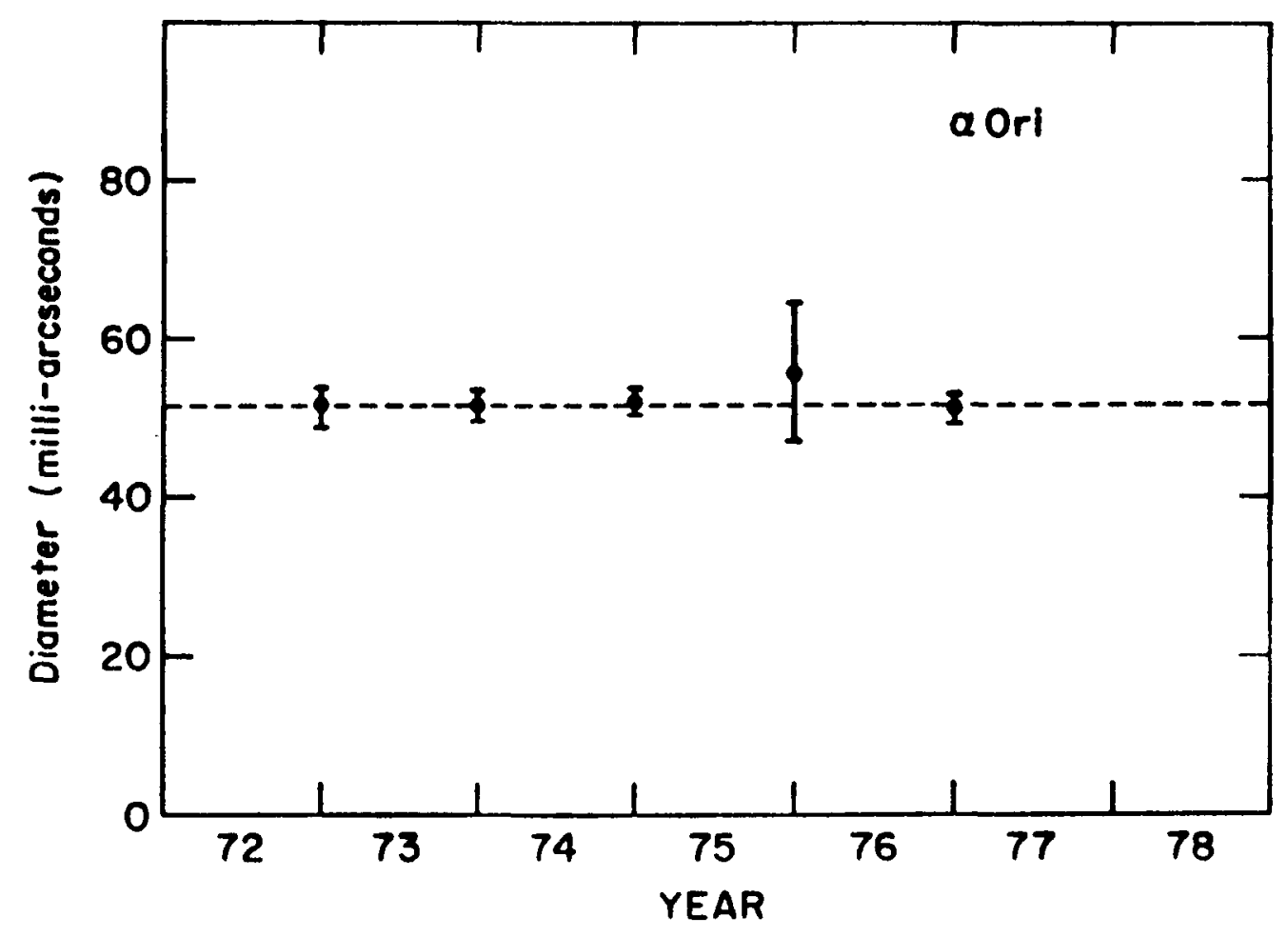

\section{Figure 9}

Measurements of the diameter of $\alpha$ Orfonis over a five year span show the reproductbility of the results of the cassegrain interferometer.

\section{Conclusion}

An Amplitude Interferometer using independent mirrors has been built at the University of Maryland. At the test site in Maryland, the interferometer has a baseline of three meters, but the instrument is designed to work in a regular observing program with baselines up to 50 meters at a permanent observing site. The design of the long baseline interferometer is based to a large extent on the design of the successful cassegrain Amplitude Interferometer and the visibility measurement method is exactly the same. The cassegrain interferometer has shown that this design produces very high values of the fringe visibility and that the results are reproducible. It has also indicated that no significant atmospheric problems should occur out to baselines of at least 50 meters. 


\section{REFERENCES}

1) D.G. Currie, Appendices II and Vili of "Woods Hole Summer Study on Synthetic Aperture Optics" (Vol. 2), Nat. Acad. Sc1. - Nat. Res. Counc11 (Wash., D.C., 1967)

2) D.G.Currie, S.L.Knapp and K.M.Liewer, Ap.J., 187, 56, 1974.

3) S.L. Knapp, D.G. Currie and K.M. Liewer, Ap.J., 198, 56, 1975.

4) K.M. Liewer, D.G. Currie and S.L. Knapp, Bu11. Am. Ast. Soc.,9, 598, 1977.

5) D.G. Currie, S.L. Knapp, K.M. Liewer and R.H. Braunstein, University of Maryland Tech. Report 非6-125, 1976.

6) K.M. Liewer, R.H. Braunstein, D.G. Currie and S.L. Knapp, Bu11. Am. Ast. Soc., 9, 598, 1977.

7) K.M. Liewer, Ph.D. Thesis, University of Maryland, 1974.

8) R.H. Braunstein, Ph.D. Thesis, University of Maryland, 1978.

\section{DISCUSSION}

P. Connes: Why is the old existing 15 metre interferometer on Mount Wilson not modernized for use by these techniques?

K.M. Liewer: The old interferometer has two serious problems:

a) beam flexure

and b) it requires very accurate guiding of the entire assembly.

These problems could be overcome but the only real advantage this instrument has over one like ours is that it does not need a moving delay line. We feel that the delay line problem is less difficult than (a) and (b) above, and our instrument has the advantage that the baseline is easily extended to much more than $15 \mathrm{~m}$. It is a shame however to see this equipment rusting away and we have wondered if perhaps one of the local amateur astronomy groups could rehabilitate it.

D.Y. Gezari: In answer to Dr. Connes' question, apparently the 50 foot interferometer has been partially cannibalized for its antique electrical parts to repair the control systems of the telescopes on Mount Wilson. It would probably be a very expensive undertaking to restore the interferometer to the point where its suitability for modern work could be determined. But it is a shame to have such a beautiful device lie idle. 